\title{
Minkish dispositions
}

\author{
Alan Hájek ${ }^{1}$
}

\section{ABSTRACT}

Start with an ordinary disposition ascription, like 'the wire is live' or 'the glass is fragile'. Lewis (1997) gives a canonical template for what he regards as the analysandum of such an ascription:

"Something $x$ is disposed at time $t$ to give response $r$ to stimulus $s$ ".

For example,

(*) the wire is disposed at noon to conduct electrical current when touched by a conductor

What Lewis calls "the simple conditional analysis" gives putatively necessary and sufficient conditions for the analysandum in terms of a counterfactual:

"if $x$ were to undergo stimulus $s$ at time $t, x$ would give response $r$ ".

Call this the counterfactual analysans. For example:

(Would-conduct) If the wire were to be touched by a conductor at noon, the wire would conduct electricity.

So we have three things in play:

(1) the ordinary disposition ascription (e.g. 'the wire is live');

(2) the canonical template (e.g. *) that is supposed to formalize this disposition ascription; and

(3) the counterfactual analysans that is supposed to provide an analysis of the canonical template (e.g. Would-conduct).

Finkish dispositions have been widely regarded as counterexamples to the adequacy of (3) as an analysis of (2). I will argue that they are not. They succeed, however, as counterexamples to the adequacy of (3) as an analysis of (1). That said, the classic cases are somewhat contrived. I will introduce the notion of a minkish disposition: a disposition that something has, even though it might not display it in response to the relevant stimulus. Cases of minkish dispositions are entirely familiar. They refute the adequacy of (3) both as an analysis of (2) and of (1). I will argue that they also refute Lewis's own, more complicated counterfactual analysis of dispositions, and bring out an internal tension in his views.

\footnotetext{
1 Many thanks to Rachael Briggs, Alex Byrne, Brian Garrett, John Hawthorne, David Manley, Jeremy Strasser, and especially John Cusbert, Yoaav Isaacs, Hanti Lin, Aidan Lyon, John Maier, Daniel Nolan, Philip Pettit, Susanna Rinard, Wolfgang Schwarz, and an audience at Trnava University and at the American Association of Philosophy (Pacific) meeting, 2015, for very helpful discussions. Further thanks to two anonymous referees for Synthese for extremely thorough and incisive comments. Research for this paper was supported in part by the Australian Research Council, Discovery Project DP130104665.
} 


\section{The simple counterfactual analysis}

Start with an ordinary disposition ascription, like 'the wire is live' or 'the glass is fragile'. Lewis (1997) gives a canonical template for what he regards as the analysandum of such an ascription:

"Something $x$ is disposed at time $t$ to give response $r$ to stimulus $s$ " (143).

Now, I think that some disposition ascriptions do not fit the canonical template. Light rays are disposed to follow geodesics simpliciter, rather than disposed to do so in response to some stimulus. ${ }^{2}$ Still, most disposition ascriptions apparently do fit the canonical template. For example,

(*) the wire is disposed at noon to conduct electrical current when touched by a conductor

or

the glass is disposed at 3:17 pm to break when struck.

What Lewis calls "the simple conditional analysis" gives putatively necessary and sufficient conditions for the analysandum in terms of a counterfactual:

"if $x$ were to undergo stimulus $s$ at time $t, x$ would give response $r$ ". (143)

Call this the counterfactual analysans. For example:

(Would-conduct) If the wire were to be touched by a conductor at noon, the wire would conduct electricity.

So we have three things in play:

(1) the ordinary disposition ascription (e.g., 'the wire is live');

(2) the canonical template (e.g., *) that is supposed to formalize this disposition ascription; and

(3) the counterfactual analysans that is supposed to provided an analysis of the canonical template (e.g. Would-conduct).

(2) and (3) are, respectively, the first and second step of what has come to be known as Lewis's “two-step” approach to dispositions (Choi 2003).

Finkish dispositions have been widely regarded as counterexamples to the adequacy of (3) as an analysis of (2). I will argue that they are not. They succeed, however, as counterexamples to the adequacy of (3) as an analysis of (1). That said, the classic cases are somewhat contrived. I will introduce the notion of a minkish disposition: a

\footnotetext{
${ }^{2}$ Fara (2005), Manley and Wasserman (2008), and Vetter (2014) give further examples of dispositions without any stimulus conditions.
} 
disposition that something has, even though it might not display it in response to the relevant stimulus. Cases of minkish dispositions are entirely familiar. They refute the adequacy of (3) both as an analysis of (2) and of (1). I will argue that they also refute Lewis's own, more complicated counterfactual analysis of dispositions.

\section{Finkish dispositions}

C. B. Martin (1994) famously introduced the notion of finkish dispositions, which have widely been thought to provide counterexamples to the counterfactual analysis of the quasi-technical canonical template. Indeed, Lewis regards it to have been "decisively refuted" (143) by Martin. For decades, the "refutation" had been "a matter of folklore", but it seems that the appearance of Martin's article finally in 1994 prompted Lewis to respond to it soon afterwards.

Martin's classic example-let us not prejudge whether it is a counterexampleinvolves a wire that is dead. However, it is

connected to a machine, an electro-fink, which can provide itself with reliable information as to exactly when a wire connected to it is touched by a conductor. When such contact occurs the electro-fink reacts (instantaneously, we are supposing) by making the wire live for the duration of the contact. (2-3).

The idea is that the counterfactual is true at the relevant time-noon, say:

if the wire were to be touched by a conductor at noon, the wire would conduct electrical current.

So the simple conditional analysis judges that $(*)$ is true. But supposing that the wire is not in fact touched by a conductor, $\left(^{*}\right)$ is false - or so we are supposed to intuit. After all, the thought goes, in that case the wire remains dead; the electro-fink is not activated.

This is regarded as providing a counterexample to the right-to-left direction of the analysis. Martin follows it with an example that is supposed to refute the left-to-right direction. Now let our wire be live. The machine is set to a "reverse cycle", so that it renders the wire dead when and only when a conductor touches it. $(*)$ is true, while the corresponding counterfactual is false - or so we are supposed to intuit. Manley and Wasserman (2008) call this a "reverse fink" case: one in which the object loses the disposition when exposed to the stimulus. Note that Lewis defines things the other way round: for him, a finkish disposition is "a disposition, which would straight away vanish if put to the test" (144). Not that it matters much: if an object has a disposition that is finkish in Lewis's sense, then it automatically lacks a contrary disposition that is finkish 
in Manley and Wasserman's sense. In the second example, the wire has the disposition of being live (which it loses upon the stimulus), which is finkish in Lewis's sense; but it lacks the disposition of being dead (which it gains upon the stimulus), which is finkish in Manley and Wasserman's sense. I will follow Lewis's usage, partly because it is more natural to speak of the dispositions - finkish or otherwise - that something has rather than lacks.

Lewis offers another example of a finkish disposition:

A sorcerer takes a liking to a fragile glass, one that is a perfect intrinsic duplicate of all the other fragile glasses off the same production line. He does nothing at all to change the dispositional character of his glass. He only watches and waits, resolved that if ever his glass is struck, then, quick as a flash, he will cast a spell that changes the glass, renders it no longer fragile, and thereby aborts the process of breaking. So his finkishly fragile glass would not break if struck. (147)

The counterfactual is false; but we are supposed to intuit that the glass is disposed to break when struck at the relevant time.

I do not.

\section{Resisting the 'counterexamples'}

Nor do I intuit as I am supposed to with respect to the canonical templates in Martin's original cases. I want to be a circuit-breaker of this current of thought in the dispositions literature.

In the first wire case, the simple conditional analysis would have us judge that $(*)$ is true. And so do I. The wire is disposed at noon to conduct electrical current when touched by a conductor. After all, its intrinsic nature is disposed to change when the electro-fink machine operates on it. ${ }^{3}$ And thanks to its intrinsic nature changing, it is then disposed to conduct electrical current; after all, it has changed into a live wire! We may easily suppose that not all wires are mutable like this: there are various other wires that the electro-fink cannot change. Our wire, on the other hand, is so responsive to the electro-fink that it can change one of its important properties at the touch of a conductor. We must take this into account when assessing what it is disposed to do when touched.

\footnotetext{
${ }^{3}$ Martin does not use the word "intrinsic" at all, but I take it that this understanding of what is going on in his case is standard. See e.g. Manley and Wasserman (2008), who are explicit about finks involving intrinsic changes in response to the relevant stimuli. For example, regarding the reverse cycle electrofink, they write: "If a conductor were to touch the wire, the fink would immediately remove the disposition to conduct electricity by altering its internal structure" (60, my emphasis). This understanding also underlies Lewis's purported solution to the problem of finks, which involves an object's retaining an intrinsic property.
} 
So when it comes to the lessons that others have drawn from the electro-fink, I am a resister.

Things are similar with the sorcerer's glass, which of course is far-fetched (not that Lewis claims otherwise). Indeed, the glass would not break if struck. But I contend that likewise it is not disposed to break if struck. Rather, it is disposed to change into a sturdy glass, thanks to the sorcerer's spell. We may suppose that not all glasses are so disposed: there are some glasses on which the sorcerer's spells are impotent. Our glass, however, is receptive to his spells; and its receptivity is a disposition that it has at the time in question. As a result, it is disposed to acquire a new disposition: to remain intact if struck. ${ }^{4}$

It is crucial to Lewis's example that the sorcerer's casting a spell is extrinsic to the glass. He insists that "dispositions are an intrinsic matter" (147). Well, are they? He immediately qualifies this parenthetically: "(Except perhaps in so far as they depend on the laws of nature.)" This seems like an important qualification to me, and it alerts us to how a thing's dispositions depend on the environment in which it finds itself - the environment perhaps encompassing the entire world. Indeed, any counterfactual analysis of dispositions makes them partly extrinsic to the things that possess them, on standard views of counterfactuals, since the truth conditions for counterfactuals depend partly on goings-on outside those things. ${ }^{5}$ On the usual Lewis-style semantics, they

\footnotetext{
${ }^{4}$ My verdict about this case is the same as Choi's (2008), but I give a reason for it that he does not give. He considers firstly a parallel case, "Bird's antidote case", in which "[a] glass $G_{1}$ is struck but does not break because, quick as a flash, a sorcerer administers an antidote that cancels out the shock of the striking and thereby aborts the process of breaking" (797). Choi then reasons as follows:

if an object is situated in a stimulating circumstance $c$ but does not exhibit a manifestation $m$ because of the masking operation of a dispositional antidote, we will deny that it has the disposition to exhibit $m$ in response to being situated in $c$; instead we will ascribe to it the disposition to exhibit $m$ in response to being situated in $c$ in the absence of the antidote. For me, therefore, $G_{1}$ is not disposed to break in response to being struck but is disposed to break in response to being struck in the absence of the sorcerer. (797-798)

He does not explain directly why we should deny that it has the disposition in question. Instead, he goes on to consider some possible reasons why we might affirm that it has the disposition, and he finds them wanting.

He turns, then, to the case that we have been considering, in which the sorcerer toughens the glass itself just before the strike (rather than administering an antidote that cancels its shock immediately afterwards). He writes: "by going through exactly the same reasoning as we did with respect to Bird's antidote case, we can easily see that there is no reason at all to believe that the glass has [the disposition to break in response to being struck]" (803). Of course, I entirely agree with Choi's denial that the glass is disposed to break in response to being struck. But this is not fully explained by his going on to say that it is disposed to break in response to being struck in the absence of the sorcerer-that's clear, but it leaves open the possibility that the glass is disposed to break in response to being struck both in the absence and in the presence of the sorcerer. I have given further support for the denial by closing off this possibility: the glass is disposed to be toughened by the sorcerer's spell. That's why it's false that it is disposed to break in the presence of the sorcerer.
}

${ }^{5}$ Manley and Wasserman's "problem of accidental closeness" (70) is a version of this point. 
depend on similarity relations among entire worlds. One can't get more extrinsic than that! Moreover, Lewis himself gives a counterfactual analysis of dispositions, so he is especially ill-placed to say that dispositions are an intrinsic matter. Changing the thing's environment - in a limiting case, the entire world in which it is embedded — can change which counterfactuals are true of it. This is certainly true of the counterfactuals that appear in Lewis's own analysis, to which we will turn later.

And so it is with the sorcerer's glass. The glass has the peculiar disposition that it has partly in virtue of being in a peculiar environment. But the fact that the disposition is sensitive to its environment is not peculiar - it is a perfectly mundane fact about many familiar dispositions. The match is disposed to light; but this disposition is hostage to extrinsic properties, such as whether its environment has oxygen or not. So I part company from Lewis when he explains what he means by "dispositions are an intrinsic matter": "if two things (actual or merely possible) are exact intrinsic duplicates (and if they are subject to the same laws of nature) then they are disposed alike" (148). The match in my oxygen-rich kitchen is disposed to light; but an intrinsic duplicate of it in a vacuum is not.

And so I say that the sorcerer's glass is disposed not to break when struck, partly in virtue of the environment that it finds itself in. Similarly, Martin's original wire is disposed to conduct when touched by a conductor, partly in virtue of the environment that it finds itself in. After all, its environment includes the electro-fink machine. In fact, we would do well to build the environment into the stimulus in Lewis's canonical template:

Something $x$ is disposed at time $t$ to give response $r$ to stimulus $s$-in-environment- $E$. (To be sure, often context will make obvious what the environment $E$ is, so it may be elided. When I am striking the match in my kitchen, it is obvious what the environment is, and that it has oxygen.) Or we might combine the environment with $x$, so that it is the system of $x$ and $E$ that has the disposition. Then the disposition might be regarded as intrinsic, I suppose, but not as a property of the thing that we were originally thinking of.

In sum, my verdicts differ from Lewis's on the truth values of

'the second wire is disposed at noon to conduct when touched by a conductor' and

'the sorcerer's glass is (now) disposed to break when struck'. 
He maintains that they are both true; I maintain that they are both false, just as the simple conditional analysis would have it.

But hang on! Isn't the reverse-fink wire live? Isn't the sorcerer's glass fragile? The cases were stipulated thus, and they appear to be coherent. The problem, I suggest, is with Lewis's canonical template (though to be fair to him, I take it to be entirely standard now). An ordinary disposition ascription should not be understood in terms of the quasitechnical schema

"Something $x$ is disposed at time $t$ to give response $r$ to stimulus $s$ ", something that we would never say outside of a philosophical context. Yes, the second wire is live; but no, it is not disposed at noon to conduct when touched by a conductor (the electro-fink sees to it that it is not). Yes, the glass is fragile; but no, it is not disposed to break when struck (the sorcerer sees to it that it is not). The simple conditional analysis does fine, for all that we have seen so far, with respect to the quasi-technical analysandum; but it fails with respect to the ordinary disposition ascription we started with. The canonical template, then, does not correctly capture the ordinary disposition ascription. And it's the ordinary disposition ascription, presumably, that we were most interested in.

\section{Minkish dispositions}

Martin supposedly gave us an example of something - a finkish live wire - that is disposed to conduct when touched by a conductor (the quasi-technical analysandum). I have just challenged this, but let us run with it now. The analysans, the counterfactual 'if the wire were touched by a conductor, it would conduct' is false. Supposedly, the contrary counterfactual is true: 'if it were touched by a conductor, it would not conduct'. But notice that this is more than is required to refute the conditional analysis. All that's required is the falsehood of the first counterfactual; that suffices to refute the left-toright direction of the analysis. Moreover, the falsehood of the first counterfactual suffices to refute the counterfactual analysis of the ordinary disposition ascription, 'the wire is live'. The truth of the contrary counterfactual is overkill.

To be sure, if the counterfactual obeys conditional excluded middle, then the falsehood of the first counterfactual entails the truth of the second. According to conditional excluded middle,

'if the wire were touched, it would conduct, 
if the wire were touched, it would not conduct'

is a tautology. So if the first disjunct is false, the second one is true. Stalnaker (1968, 1981) defends conditional excluded middle. But Lewis (1973) and various other authors deny it, and so do I. Be that as it may, what matters is the falsehood of the first disjunct, whatever the status of the second.

Similarly, in Lewis's sorcerer case, supposedly the glass is disposed to break when struck; but the counterfactual 'if the glass were struck, it would break' is false. Furthermore, 'if the glass were struck, it would remain intact' is supposed to be true. But all that matters is that the first counterfactual is false; that suffices to refute the conditional analysis - both of the quasi-technical analysandum, and of the ordinary disposition ascription 'the glass is fragile'.

Once we see this, we can see that finkish cases are unnecessarily baroque. Perfectly ordinary cases will do. Indeed, they are widespread, and even ubiquitous, as I will argue. $^{6}$ Take a perfectly ordinary fragile glass in a perfectly ordinary environment. It is disposed to give the response of breaking to the stimulus of being struck. Consider the counterfactual

'if the glass were struck, it would break'.

There are two straightforward ways in which this counterfactual may turn out false.

\section{1) Chanciness of the response}

The first straightforward way is for it to be a chancy matter whether the glass breaks when struck. Let us suppose that the chance is high, but still it has some non-negligible positive chance of surviving a given striking, but it is not struck. Then, I submit, it is false that if it were to be struck, it would break. Imagine that the chance of breaking is $90 \%$, say. (Handle with care!) But if the glass were struck, there would be a $10 \%$ chance that it would survive. This is incompatible with the claim that the glass would break, if struck. The counterfactual is second-guessing the result of a chancy process, with nonnegligible chances each way. It is the very nature of such a process that there is no fact of the matter of how it would turn out. ${ }^{7}$

Not everyone will accept this argument. A fan of conditional excluded middle, for example, might say that there is a fact of the matter; it's just that we can't determine

\footnotetext{
${ }^{6}$ Others have given examples of ordinary cases — e.g. Fara 2005 — but I am not aware of anyone having argued just how prevalent they are.

7 See Hawthorne (2005) for a closely related argument, and Williams (2008) for a response.
} 
what it is. Then let's make the process actual, so that all the facts are apparent. Suppose that in fact the glass is struck, and it survives. It is false that if it were struck, it would break; it was, and it didn't. (Here I only assume that the counterfactual obeys weak centering, advocated by Stalnaker, Lewis, and various other authors.) Yet that is consistent with its being fragile. We could rightfully say that it was lucky: the chance of its breaking was high, and yet it didn't. It is disposed to give the response of breaking to the stimulus of being struck, but the counterfactual is false. The refutation of the simple conditional analysis is that simple.

\section{2) Unspecificity of the stimulus}

The second straightforward way in which the counterfactual may turn out false is for the fragile glass to have a small spot of strength — strong enough that if it were struck there, it would survive. (This is very close to what Manley and Wasserman 2008 call a "reverse Achilles heel", a reference to Achilles' one point of weakness, although my spot of strength does not need to be quite as precise as the one in their example. ${ }^{8}$ ) Then it is false that if the glass were struck, it would break. It might be struck on its spot of strength; and in that case, it would survive. Note that there need not be anything farfetched about its being struck on its spot of strength. In the parlance of the usual similarity semantics for counterfactuals, such strikes may well take place in worlds just as similar as strikes that miss the spot of strength. Then among the closest strike-worlds, there are worlds in which the glass survives. The glass is fragile; it is disposed to give the response of breaking to the stimulus of being struck; but the corresponding counterfactual is false. This is the case on Lewis's (1973) semantics for counterfactuals (given what I have assumed about the closeness ordering); but more than that, I think that it is intuitively the case.

Again, let's now make the hypothetical case actual. The glass is fragile; it was struck; and it did not break. For as it happens, it was struck on its spot of strength. The counterfactual is false. This is the case as long as the counterfactual obeys weak centering; but more than that, I think that it is intuitively the case.

\footnotetext{
${ }^{8}$ They say of an "incredibly delicate crystal glass" that "it can withstand a surprisingly strong force, provided that the force is applied at exactly the right angle and at exactly the right point. Despite the reverse Achilles' heel, the glass is extremely fragile" (69). Their italics suggest that their "reverse Achilles heel" is extremely localised. My fragile glass's spot of strength could be bigger than that -it might be, say, $10 \%$ of the glass. To be sure, when they later come to their own analysis, they write: "We can then say that something is disposed to break when dropped if and only most dropping cases are such that the object would break in them" (75), which clearly allows for larger regions of strength on a fragile glass.
} 
I have said both here and in the chancy cases that the counterfactual is false. But perhaps instead you think that it is indeterminate. Perhaps you think that there is no fact of that matter of what the chancy glass would do if it were struck, or of whether the striking would hit or miss its spot of strength, when the striking is hypothetical. That will not save the counterfactual analysis. For it is true that the glass is fragile, and true that it is disposed to break if struck - not indeterminate. In any case, in the actual (as opposed to hypothetical) cases, all the facts are in: the counterfactuals are false, not indeterminate.

Note that the first straightforward way in which the counterfactual may be false (or indeterminate) does not reduce to the second. It may be that all ways of striking the glass yield its breaking with a fixed high chance.

Nor does the second way reduce to the first. It may be that the glass's chance of breaking would be very close to 0 if the glass were struck on its spot of strength, while anywhere else the chance would be very close to 1 . Then 'if the glass were struck, the chance of it breaking would be high (e.g. 90\%)' is false; it would be low if the spot of strength were struck. This shows that we cannot simply make the natural small amendment to the simple conditional analysans:

"if $x$ were to undergo stimulus $s$ at time $t, x$ would probably give response $r$ ". 9

So far I have treated the two ways separately, but we may combine them to create yet more trouble for the simple conditional analysis. Now suppose that the glass is extremely delicate everywhere except for a small spot of chancy strength. If it were to be struck anywhere but that spot, it would certainly break; if it were to be struck on the spot, it would very probably break, but not certainly do so - the chance of its doing so would be, say, 90\%. Still, 'if the glass were struck, it would break' is false: it might be struck on its point of chancy strength; and in that case, it might survive (the chance of its doing so would be $10 \%) .{ }^{10}$

\footnotetext{
9 There is a further concern regarding the notion of probability here. It is presumably some sort of objective chance. The propensity interpretation is one of the leading candidates for understanding that. But propensities are often regarded as graded dispositions. Then the amended analysis is in danger of being circular: analysing a disposition in terms of a (graded) disposition. (Thanks here to Jeremy Strasser.)

${ }^{10}$ Note that Manley and Wasserman's (2008) reverse Achilles cases are stronger than are needed to be trouble for various conditional analyses. For example, they consider "a possible glass with a reverse Achilles' heel that would fail to break in exactly [a given fully precise dropping] scenario" (69, my emphasis). It suffices that the glass might fail to break in that scenario.
} 
Yet again, let's now make the hypothetical case actual. The glass is fragile; it was struck; and it did not break. For as it happens, it was struck on its spot of chancy strength; and by (small) chance, it survived that striking. The counterfactual is still false.

These two straightforward ways in which the counterfactual analysans can go false (or indeterminate), either working individually or in tandem, generalise to other dispositions. The first way generalises to dispositions that are not sure-fire. But in the chancy world that we live in, those may well be most dispositions - quantum mechanical indeterminism percolating up to macroscopic objects makes this plausible. (Cf. Hawthorne 2005.) Indeed, fragility is as paradigmatic a case of a disposition as there can be-it is by far the most common example in the literature-and yet given its sensitivity to facts about molecular bonding, it is surely not sure-fire (even when it is nearly so). So it will not do to restrict ourselves to analysing sure-fire dispositions, pretending that our job is done.

Moreover, we may want to allow that things can have dispositions even when they fall far short of being sure-fire. Taking a lead from the literature on generics, we might call something a disposition even when it is less likely to fire than not to fire. We might say that rattlesnakes are disposed to attack when provoked, even if the chance of a rattlesnake attack upon provocation is less than $1 / 2 .{ }^{11}$ It could be a true thing to say in a context in which rattlesnakes are contrasted to other snakes that are much more docile. In fact, we may be able to push this thought to the extreme (though I admit that this is more easily contested). A uranium-238 atom is highly radioactive: it is disposed to decay at every moment. (It seems false to say that it has no disposition to decay at a given moment, especially in a context in which we are contrasting it with stable atoms such as gold.) But the chance of the uranium atom decaying at a given moment is $0 .{ }^{12}$

The second straightforward way exploits any lack of precision in the antecedent of the dispositional counterfactual, precisifying it with a realisation of the stimulus in which it's not the case that the response would be given. Take your favorite disposition of a familiar kind of object, with its associated stimulus. I bet I could come up with an ordinary instance of that object — not a finkish one - that has the disposition, but for which some particular instantiation of the stimulus would fail to yield the response. And we may combine the ways, as in the case of the glass with the chancy spot of strength:

\footnotetext{
${ }^{11}$ See Manley and Wasserman (2008) for further such examples.

12 Thanks to Hanti Lin for this example.
} 
precisifying with a realisation of the stimulus in which it's not guaranteed that the response would be given. This covers still more problematic cases, many of which will be perfectly mundane.

I think of both straightforward ways of showing the counterfactual analysans to be false in terms of a contrary might counterfactual. Consider the glass whose breaking is chancy. If you were to strike it, it might not break-it might survive by chance. Consider the glass that has a spot of strength-even merely a spot of chancy strength. If you were to strike it, it might not break - it might be struck on that spot. Either way, it is false that if you were to strike it, it would break. More generally, I regard 'might not' counterfactuals to be incompatible with the corresponding 'would' counterfactuals. ${ }^{13}$ So does Lewis (1973); in fact, he upholds the stronger thesis that 'would' and 'might' counterfactuals are duals. ${ }^{14}$ Hence, I think that he should be favorably disposed to my counterexamples.

So we have an easy recipe for counterexamples to the simple conditional analysis: $x$ is disposed at time $t$ to give response $r$ to stimulus $s$, but $x$ might not give response $r$ at time $t$ to stimulus $s$. Let us call this a minkish disposition. The ' $m i$ ' in 'minkish' is meant to be suggestive of the ' $m i g h t$ ' clause. Minkish dispositions, unlike finkish dispositions, are ubiquitous. The wine glass on the table beside me has a minkish disposition to break when struck (at any time): it is disposed to do so, but it might not. I'm tempted to say that the glass both has a disposition to break, and a capacity not to. I think of a minkish disposition, then, as the combination of a disposition and a contrary capacity. But I don't want to get embroiled in another debate on the nature of capacities, so I'm happy to stop at my official definition of a minkish disposition.

\footnotetext{
${ }^{13}$ This is one reason why I regard the chancy counterfactuals and unspecific-antecedent counterfactuals to be false, rather than indeterminate.

14 In (1986, pp. 63-64) he considers another reading of 'might' counterfactuals, a 'would-be-possible' reading. 'If it were the case that $p$, it might be that $q$ ' is analysed as 'if it were the case that $p$, it would be the case that: $q$ is possible'. The trouble with this reading of the 'might' counterfactuals that I have in mind is that it does not do justice to the conflict between 'would' and the corresponding 'might not' counterfactuals. I called it the conflict of incompatibility. Perhaps I am wrong, and it is not as bad as that; but we should all agree that there is still tension between them. (DeRose 1999 gives a pragmatic explanation of the tension, while calling it an "inescapable clash".) But on the 'would-be-possible' reading, there should be no tension at all— just as there is no tension at all between $p$ and possibly not-p. 'I am a philosopher, but it is possible for me not to be a philosopher' glides smoothly off the tongue.
} 


\section{More monkeying with minkishness}

Some other authors have pointed out problems that chancy-response dispositions pose for certain counterfactual accounts of dispositions. For example, Vetter (2011) does so for Manley and Wasserman's (2008) account, taking as her point of departure Hawthorne's (2005) argument that chanciness threatens the usual similarity-semantics for counterfactuals with becoming an error theory. And the problem of unspecific stimuli has also been noticed. Manley and Wasserman, for their part, deploy it against certain conditional analyses of dispositions. ${ }^{15}$ The notion of minkish dispositions, then, unifies these two strands in the literature: they both involve cases in which a disposition's response might not be displayed upon a stimulus of the requisite kind.

In fact, minkish dispositions arguably go even further. For a response might not be displayed even when its chance is 1 - think of the uranium atom's disposition not to decay at a given time, which might not be displayed even though its chance is $1 .{ }^{16}$ Or consider cases that conform to the stimulus-response canonical template. Suppose that a fair coin is tossed infinitely often. It might land heads every time, although the chance of its doing so is 0. (See Williamson 2007.) So it seems that the counterfactual 'if the coin were tossed infinitely often, it would land tails at least once' is false, despite its consequent having chance 1 given its antecedent. For it is incompatible with 'if the coin were tossed infinitely often, it might not land tails', which is true. Yet we should say that the coin is disposed to land tails at least once upon being tossed infinitely often. Another example: consider a fair (unbiased) throw of an infinitely thin dart at a representation of the real-valued interval $[0,1]$. The dart is disposed to hit an irrational number upon being thrown - it does so with chance 1. But it might hit a rational number, despite the set of such numbers having measure 0 . So the simple-analysis counterfactual is false: 'if the dart were thrown, it would hit an irrational number'. More generally, possibility and probability do not mesh well. Probability 0 does not entail impossibility; probability 1 does not entail necessity.

On such a liberal notion of what might happen, it would seem that virtually every disposition is minkish, and hence that the simple conditional analysis hardly gets any cases right! But we need not go that far for minkish dispositions to earn their

\footnotetext{
${ }^{15}$ See also Choi (2011) for a response to Manley and Wasserman and defense of the simple conditional analysis.

${ }^{16}$ I am assuming that the atom can have contrary dispositions, which differ in degrees: a weak disposition to decay at any given time, and a strong disposition not to do so.
} 
philosophical keep. It is enough that they render false the analysis's counterfactuals for entirely familiar disposition ascriptions to entirely familiar things, such as the wine glass on the table beside me. ${ }^{17}$

Minkish dispositions have three dialectical advantages over finkish dispositions. Firstly, minkish dispositions are not remotely contrived or unusual. I suspect that most, and perhaps even all, fragile glasses are minkishly fragile! Secondly, I have argued that finkish dispositions do not provide counterexamples to the simple conditional analysis of the Lewisian analysandum, the quasi-technical canonical template. Minkish dispositions do. They are counterexamples to the adequacy of the counterfactual treatment of both the canonical template and the ordinary disposition ascription. Unlike finkish dispositions, minkish dispositions drive a wedge between disposition ascriptions and counterfactuals. Thirdly, unlike finkish dispositions, minkish dispositions provide counterexamples to various sophisticated counterfactual analyses of dispositions; I turn now to Lewis's analysis. ${ }^{18}$

\section{Lewis's analysis}

Lewis himself calls his "reformed conditional analysis" an "unlovely mouthful". Here it is:

${ }^{17}$ In any case, I disagree with Choi $(2008,11)$ when he writes: "it is not objectionable at all that it is conceptually impossible that a bearer of [the disposition to break in response to being struck] is struck but does not break"; indeed, he regards this as an advantage of the simple conditional analysis. More generally, he regards it as an advantage that "[i]n general, according to [the simple conditional analysis], $x$ 's being disposed to exhibit $m$ in response to being situated in $c$ entails that $x$ exhibits $m$ if situated in $c$ " (11, my emphasis). But consider what I would describe as three extremely fragile glasses:

1) The first shatters with probability 1 when hit, but nonetheless it is not guaranteed to do so. (Probability 1 does not entail necessity, remember!)

2) The second has exactly a point of strength; if it were to be hit anywhere but this set of measure zero, it would shatter.

And even more fragile still:

3) The third is guaranteed to shatter when hit anywhere apart from exactly one point; and even if hit there, it would shatter with probability 1 , but nonetheless it is not guaranteed to do so.

In each case, it is conceptually possible that the glass is struck but does not break. So by Choi's lights, none of these glasses are fragile. I must say I find that conclusion objectionable.

I think it is far more natural to allow, as Manley and Wasserman do, that dispositions come in degrees; when the degree is sufficiently high for a given entity, we may truly predicate the disposition of the entity. (As I observed with the rattlesnake and uranium decay examples, in some contexts the response need not even have high probability, let alone being necessitated.) Be that as it may, the entailments that Choi countenances will hardly ever hold, if only because of the possibility of extremely low-chance quantum mechanical anomalies. He seems to be committed to an error theory about dispositions. (Cf. Hawthorne 2005, again.)

${ }^{18}$ Other such analyses include those of Manley and Wasserman (2008) and Choi (2008, 2012). It should be clear that minkish dispositions are also problematic for their analyses. (I have cited Vetter (2011) in connection with Manley and Wasserman's analysis; see footnote 17 for cases that are problematic for Choi's.) However, my main such target in this paper is Lewis's analysis. 
Something $x$ is disposed at time $t$ to give response $r$ to stimulus $s$ iff, for some intrinsic property $B$ that $x$ has at $t$, for some time $t^{\prime}$ after $t$, if $x$ were to undergo stimulus $s$ at time $t$ and retain property $B$ until $t^{\prime}, s$ and $x^{\prime}$ s having of $B$ would jointly be an $x$-complete cause of $x$ 's giving response $r$. (157)

This is a lot to take in at once; he works up to it in stages. Dropping the reference to time for now, the rough idea is that $x$ is disposed to give response $r$ to stimulus $s$ just in case: $x$ has some intrinsic property such that, if it were retained, it and the stimulus would cause $x$ to give response $r$. Think of the property as the causal basis for the response. The fragile glass has such a property-its molecular structure, presumably. If the glass were to retain this structure and be struck, it would break. The sorcerer's finkish glass is no counterexample, because its molecular structure changes when it is struck (presumably); the sorcerer's spell ensures this.

Lewis adds complications to this rough idea. He wants to allow that a bit of time could elapse between the onset of the stimulus and the vanishing of a finkish disposition, so he builds into his analysis two moments of time. He also wants to allow for the possibility of a finkish partial lack of a causal basis.

The glass has property $B$ but it lacks property $B^{\prime} . B$ and $B^{\prime}$ together would constitute a causal basis for breaking if struck; that is, striking and having $B$ and having $B^{\prime}$ would together cause breaking. $B$ alone is not a causal basis: striking and having $B$ would not suffice to cause breaking. But the lack of $B^{\prime}$ is a finkish lack. If the glass were struck, straight away it would gain $B^{\prime}$; and in addition it would retain $B$; and so it would break. And $B$, together with the striking, would be a cause of the breaking. Not, indeed, the complete cause; but a part of the cause is still a cause, so our analysans in its present form is satisfied. And yet because of the lack of $B^{\prime}$ it seems false that the unstruck glass is fragile. (156)

This adverts to the notion of a "complete cause". Lewis introduces "a restriction of that notion: a cause complete in so far as havings of properties intrinsic to $x$ are concerned, though perhaps omitting some events extrinsic to $x$. For short, 'an $x$-complete cause"”.

All of the materials for Lewis's analysis, then, are in place. But Choi and Fara (2014, with small changes in their notation) give a helpful simplification of it: "An object $x$ is disposed to $R$ when $S$ iff $x$ has an intrinsic property $B$ such that, if it were the case that $S$, and if $x$ were to retain $B$ for a sufficient time, then $S$ and $B$ would jointly cause $x$ to $R$." It will be easier to see how my argument targets this version. But it should be clear that the unsimplified Lewis version will be vulnerable in just the same way-it is before you so that you can check. 


\section{Striking at Lewis's analysis}

I am by no means the first to offer objections to Lewis's analysis—see, for example, Bird (1998), Choi (2005), and Handfield (2008). However, I believe that the objections that I will offer now against that analysis are new. In particular, they are based on distinctly Lewisian philosophical positions, so he would be especially hard-pressed to resist them. They bring out what I take to be an internal tension in his writings.

Again, minkish dispositions provide counterexamples to his analysis. Consider a fragile glass whose fragility is not quite sure-fire: the chance of its breaking when struck is high, but not 1 . The analysandum is true: the glass is disposed to break (at any time) when struck. But the analysans is false: there is no intrinsic property of the required kind, for there is no property that together with a striking would jointly cause the glass to break. After all, there is a chance of the glass not breaking; and in that case, nothing causes its breaking. Causation is factive in both its relata: if $C$ causes $E, C$ and $E$ both occur. If there is no breaking, then there is no cause of breaking.

The easiest way to see the problem is with the lucky survival of an actual striking. Suppose that in fact the fragile glass is struck, and by chance it survives. Nothing causes it to break. A fortiori, the glass has no intrinsic property such that the property and the striking jointly cause it to break. A fortiori, the glass has no intrinsic property retained for a sufficient time such that the property and the striking jointly cause it to break. Yet the glass is fragile; that's what makes its survival lucky. ${ }^{19}$

Next, let's exploit the unspecificity of the stimulus, the striking. A glass with a spot of strength - even a chancy spot of strength - provides another counterexample. If it were to be struck, it might be struck on its spot of strength and survive. In that case, nothing would have caused it to break. Still less would some intrinsic property of the

\footnotetext{
${ }^{19}$ Kvanvig (1999) also regards probabilistic dispositions as problematic for Lewis's analysis, but in a different way. He presents a related problem first:

... given Lewis's identification of causes and counterfactuals of a certain sort, he cannot account for the dispositions of free persons. I'm disposed to eat most anything chocolate, but the counterfactual posited in Lewis's theory of causation will turn out false if that theory of causation is correct and I am free in the anti-deterministic sense. Whether or not I am so disposed, however, should not depend on whether I am free in this sense.

This difficulty arises again for any merely probabilistic disposition. Examples concerning the dispositions of radioactive material to decay pose the same kind of difficulty for any counterfactual theory of dispositions combined with a counterfactual theory of (deterministic) causation. (p. 74)

The problem that I have raised has nothing to do with Lewis's theory of causation, or indeed with any counterfactual theory of causation. All I assume is the factivity of causation, which is a platitude.

In any case, radioactive decay is another example that does not fit the Lewisian canonical template in the first place. Radioactive atoms have various dispositions to decay simpliciter, rather than in response to a stimulus.
} 
glass and the striking jointly cause the breaking. Still less would some intrinsic property of the glass retained for a sufficient time, and the striking, cause it to break.

Now suppose that the glass is actually struck on its spot of strength, and it survives. Nothing caused it to break, because it didn't. Still less did some intrinsic property of the glass and the striking jointly cause it to break. Still less did some intrinsic property of the glass retained for a sufficient time, and the striking, cause it to break.

Now we generalize. Minkish dispositions are all around us. On the Lewis analysis, surprisingly few things would be fragile - or agile, or tough, or gruff, or soluble, or voluble, or solid, or stolid, ...

\section{Minkish dispositions and Lewis's positions}

We can see why Lewis would have been drawn to the simple conditional analysis. Recall his doctrine of "Humean Supervenience": "the thesis that the whole truth about a world like ours supervenes on the spatiotemporal distribution of local qualities" (1994, 473). Dispositions are part of the whole truth about our world, so they had better supervene on the Humean mosaic of local qualities. Lewis felt that he had an account of how counterfactuals supervene on the mosaic. The conditional analysis of dispositions, then, was congenial to his metaphysical picture. ${ }^{20}$

Then along came finkish dispositions, and this happy situation was threatened. He could not tolerate having dispositions as primitives. So he set out to offer an alternative analysis of them, again in terms of counterfactuals - explicitly in the "if ... would" locution in his analysans, implicitly in the appeal to the notion of an $x$-complete cause. For that notion is reducible by Lewis's lights to the notion of intrinsic properties, and that of causation, which in turn is reducible to certain patterns of counterfactuals.

The irony is that Lewis's own distinctive approach to counterfactuals, coupled with some other central Lewisian doctrines, appear to undermine his own account of dispositions. According to his analysis of causation (1973b), causation is factive in both its relata. He believed in objective chance as a feature of the world, independently of us (1980, 1994). Moreover, he regarded it to be a pervasive feature. Now, I'm not aware of him giving so explicitly the argument that chanciness undermines counterfactualsthat a chance of alternative outcomes undermines a counterfactual that claims that a particular outcome would occur. But he comes close to giving it. See, for example, his

20 I thank Wolfgang Schwarz for drawing my attention to this. 
(1981, reprinted 1986) discussion of "full patterns" of counterfactuals that constitute “dependency hypotheses"-maximally specific propositions about how what an agent cares about does and does not depend causally on her present acts. He writes: "If the world is the chancy way I mostly think it is, there's nothing at all arbitrary or indeterminate about the counterfactuals in full patterns. They are flatly, determinately false." (331)

This is one argument that Lewis gives against conditional excluded middle (a consequence of Stalnaker's 1968 semantics for conditionals, in which his selection functions pick out unique antecedent-worlds). In the same passage, Lewis gives another argument that turns on counterfactuals with unspecific antecedents going false: "surely some questions about how things would be if $X$ have no nonarbitrary answers: if you had a sister, would she like blintzes?" This resonates with one of his main arguments that a similarity semantics for counterfactuals should allow for ties in which antecedentworlds are most similar to actuality. He wants to allow that both

'if Bizet and Verdi were compatriots, Bizet would be Italian' and

'if Bizet and Verdi were compatriots, Bizet would not be Italian' are false. He writes:

However little there is to choose for closeness between worlds where Bizet and Verdi are compatriots by both being Italian and worlds where they are compatriots by both being French, the selection function must choose. I do not think it can choose - not if it is based entirely on comparative similarity, anyhow. $(1973,80)$

Like the counterfactuals about Bizet and blintzes, the counterfactuals Lewis uses to analyze minkish dispositions will often go false thanks to ties among the closest worlds that realize their antecedents. ${ }^{21}$

Notice how Lewis's two arguments against conditional excluded middle correspond to the two straightforward ways in which minkish dispositions undermine

\footnotetext{
${ }^{21}$ Shortly after the passage that I cited in footnote 14, Lewis writes: "If we want any kind of similarity theory of counterfactuals, we dare not treat "there would be some chance of it" and "it would not happen" in general as incompatible" (1986, p. 65) and he goes on to give some arguments for their compatibility. But this undermines one of his main arguments against conditional excluded middle, from chanciness. He can no longer say that, for example,

'if the coin were tossed it would land heads OR if the coin were tossed it would land tails' is false in virtue of the chanciness of the coin rendering each disjunct false. His other main argument against conditional excluded middle, from unspecific antecedents (“... no nonarbitrary answers") is similarly undermined by parallels to his compatibility arguments. And yet his denial of conditional excluded middle is one of the most distinctive parts of his theory of counterfactuals. I do not know how to resolve this tension in his writings - nor the tension of some of them with his own account of dispositions.
} 
counterfactuals about their realization: chanciness of the response, and unspecificity of the stimulus.

Finally, as I have noted, Lewis championed the duality of 'would' and 'might' counterfactuals. The 'would' counterfactuals of his analysis are incompatible with corresponding 'might not' counterfactuals; but for minkish dispositions, the 'might not' counterfactuals are true.

I think that Lewis would have been receptive to minkish dispositions; he just wouldn't have liked the fact that they break his account.

School of Philosophy

Australian National University

\section{REFERENCES}

Bird, Alexander (1998): “Dispositions and Antidotes”, Philosophical Quarterly 48 (191), 227-234.

Choi, Sungho (2003): “Improving Bird's Antidotes", Australasian Journal of Philosophy, 81, 573-80.

Choi, Sungho (2005): "Dispositions and Mimickers", Philosophical Studies 122, 183188.

Choi, Sungho (2008): “Dispositional Properties and Counterfactual Conditionals", Mind $117,795-841$.

Choi, Sungho (2011): “What is a Dispositional Masker?” Mind 120, 1159-1171.

Choi, Sungho (2012): “Intrinsic Finks and Dispositional/Categorical Distinction”, Noûs $46(2), 289-325$.

Choi, Sungho and Fara, Michael (2014): "Dispositions", The Stanford Encyclopedia of Philosophy (Spring 2014 Edition), Edward N. Zalta (ed.), URL = <http://plato.stanford.edu/archives/spr2014/entries/dispositions/>.

DeRose, Keith (1999): “Can It Be That It Would Have Been Even Though It Might Not Have Been?", Philosophical Perspectives 13, Epistemology, 385 -413.

Fara Michael (2005): “Dispositions and Habituals”, Noûs 39 (1), 43-82.

Handfield, Toby (2008): “Unfinkable Dispositions”, Synthese 160 (2), 297-308.

Hawthorne, John (2005): "Chance and Counterfactuals", Philosophy and Phenomenological Research 70 (2), 396-405.

Kvanvig, Jonathan L. (1999): "Lewis on Finkish Dispositions", Philosophy and Phenomenological Research 59 (3), 703-710. 
Lewis, David (1973): Counterfactuals, Basil Blackwell.

Lewis, David (1973b): “Causation”, Journal of Philosophy 70, 556-67. Reprinted in his (1986).

Lewis, David (1980): “A Subjectivist's Guide to Objective Chance”, in Studies in Inductive Logic and Probability, Vol II., University of California Press. Reprinted in his (1986).

Lewis, David (1981): "Causal Decision Theory", Australasian Journal of Philosophy 59, 5-30. Reprinted in Lewis (1986).

Lewis, David (1986): Philosophical Papers Volume II, Oxford: Oxford University Press.

Lewis, David (1994): “Humean Supervenience Debugged”, Mind 103, 473-490.

Lewis, David (1997): "Finkish Dispositions”, The Philosophical Quarterly 47, 143-158.

Manley, David and Ryan Wasserman (2008): "On Linking Dispositions and Conditionals", Mind 117, 59-84.

Martin, C.B., 1994, "Dispositions and Conditionals, The Philosophical Quarterly 44, $1-8$.

Stalnaker, Robert (1968): "A Theory of Conditionals", in N. Rescher (ed.), Studies in Logical Theory, Oxford: Blackwell.

Stalnaker, Robert (1981): “A Defense of Conditional Excluded Middle", in Ifs: Conditionals, Belief, Decision, Chance, and Time, eds. W.L. Harper, R. Stalnaker, and G. Pearce, Dordrecht: Reidel, 87-104.

Vetter, Barbara (2011): “On Linking Dispositions and Which Conditionals?”, Mind 120 (480), 1173-1189.

Vetter, Barbara (2014): “Dispositions Without Conditionals”, Mind 123 (489), 129-156.

Williams, J. Robert G. (2008): “Chances, Counterfactuals, and Similarity”, Philosophy and Phenomenological Research 77 (2), 385-420.

Williamson, Timothy (2007): "How Probable Is an Infinite Sequence of Heads?" Analysis 67, 173-80. 